\title{
GENERIC STABILITY PROPERTIES OF PERIODIC POINTS
}

\author{
BY \\ K. R. MEYER( ${ }^{(1)}$
}

\begin{abstract}
A classification of the periodic points of a generic area-perserving diffeomorphism which depends on a parameter is given. The stability properties of each periodic point in the classification is decided.
\end{abstract}

1. Introduction. This paper is a sequel to [3]. In the previous paper a classification of the periodic points of a generic area-preserving diffeomorphism which depends on a parameter was given. The bifurcation or branching of these periodic points was analyzed in detail. Here the stability properties of these periodic points will be analyzed.

The transversality methods developed by Thom and others gives information only about the germ of a generic map and not about the map itself. Often a standard theorem from analysis can be applied to yield information about the map. Indeed this was the case for several of the types of periodic points in the classification but for several others the existing literature was not sufficient. In order to establish the stability of a periodic point the invariant curve theorem of Moser [4] is used and in order to establish the instability of a periodic point a Liapunov function is constructed. The stability or instability of each type of periodic point in the classification is established.

Further discussion on the origin of the present problem in the theory of Hamiltonian differential equations can be found in [3].

2. Statement of the main theorem. Recall that $\mathscr{F}$ denotes the space of all smooth maps $\varphi: M \times S \rightarrow M$ such that $\varphi_{s}=\varphi(\cdot, s): M \rightarrow M$ is a sympectic diffeomorphism for each $s \in S=S^{1}$ where $M$ is a compact, smooth, two-dimensional symplectic manifold. $\mathscr{F}$ has the topology of $C^{\infty}$ maps and hence is a Baire space. A point $(x, s) \in M \times S$ is called a periodic point of least period $m$ if $\varphi_{s}^{m}(x)=x$ and $m$ is the least positive integer for which this relation holds. The eigenvalues of $d \varphi_{s}^{m}(x)$ are called the multipliers of $(x, s)$.

In [3] a residual set $\mathscr{G} \subset \mathscr{F}$ was defined such that if $(x, s)$ is a periodic point of $\varphi \in G$ then either it is (1) an extremal or (2) a transitional or (3) a hyperbolic or

Received by the editors March 13, 1970.

AMS 1969 subject classifications. Primary 3465; Secondary 3440, 3451, 5750.

Key words and phrases. Generic, Baire category, area-preserving, symplectic, stability, periodic point.

(1) This research is supported by ONR contract number NONR 3776(00). 
(4) an elliptic periodic point. If $\lambda, \lambda^{-1}$ are the multipliers of $(x, s)$ then roughly speaking the classification is (1) $\lambda=+1$, (2) $\lambda=-1$, (3) $\lambda \neq \pm 1, \lambda$ real, (4) $\lambda \neq \pm 1, \lambda$ complex and $|\lambda|=1$. Further conditions are placed on the higher derivatives of $\varphi$ in the first two cases, and the reader is referred to [3] for the complete definitions of these terms. Furthermore if $(x, s)$ is an elliptic periodic point whose multipliers are $k$ th roots of unity then additional conditions were imposed on the higher derivatives and such a point was called a $k$-bifurcation point.

Here we shall further restrict those elliptic points which are not $k$-bifurcation points. Let $(x, s)$ be a periodic point of least period $m$ of $\varphi \in \mathscr{F}$ with multipliers $\exp ( \pm 2 \pi \omega i)$ where $\omega$ is not a fraction of the form $a / b, 0<a<b \leqq 8$. Then by Birkhoff's normalized procedure [1] there exists a symplectic chart $(q, p)$ at $x, q(x)$ $=p(x)=0$, and a chart $\varepsilon$ at $s, \varepsilon(s)=0$, such that $\varphi^{m}:(q, p, \varepsilon) \rightarrow(Q, P)$ where

$$
\begin{array}{ll}
q=r^{1 / 2} \cos \theta, & p=r^{1 / 2} \sin \theta, \\
Q=R^{1 / 2} \cos \Theta, & P=R^{1 / 2} \sin \Theta,
\end{array}
$$

and

$$
\begin{aligned}
& \Theta=\theta+2 \pi \omega+\varepsilon \alpha(\varepsilon)+\beta_{1}(\varepsilon) r+\beta_{2}(\varepsilon) r^{2}+\Theta_{1}(\theta, r, \varepsilon), \\
& R=r+R_{1}(\theta, r, \varepsilon),
\end{aligned}
$$

with all displayed functions smooth in $\theta, r^{1 / 2}, \varepsilon$ and $2 \pi$ periodic in $\theta$. Moreover $\Theta_{1}=O\left(r^{3}\right)$ and $R_{1}=O\left(r^{4}\right)$.

Definition 2.1. The periodic point $(x, s)$ will be called a general elliptic periodic point if $\omega$ is irrational and $\beta_{1}(0)^{2}+\beta_{2}(0)^{2} \neq 0$.

Note that the definition of general elliptic point is slightly different from that found in many references since the first twist coefficient $\beta_{1}(0)$ may be zero. One cannot be assured that $\beta_{1}$ is always nonzero when the map depends on a parameter. The above definition is adequate for the application of the invariant curve theorem to establish stability.

Definition 2.2. Let $\mathscr{H} \subset \mathscr{F}$ be the set of all $\varphi \in \mathscr{F}$ such that if $(x, s)$ is a periodic point of $\varphi$ then $(x, s)$ is either (1) an extremal or (2) a transitional or (3) a hyperbolic or (4) a $k$-bifurcation or (5) a general elliptic periodic point.

Clearly $\mathscr{H} \subset \mathscr{G}$ and the only new requirement is (5).

The first theorem of this paper is a minor extension of the main theorem of our previous paper, namely

\section{THEOREM 2.3. $\mathscr{H}$ is residual in $\mathscr{F}$.}

In the analysis of transitional periodic points [3, Proposition 1.13], and 4bifurcation points [3, Proposition 1.17], two additional subcases were considered called cases (A) and (B). The main theorem of this paper is

THeORem 2.4. A periodic point of $\varphi \in \mathscr{H}$ is stable if it is either general elliptic; $k$-bifurcation, $k \geqq 5$; 4-bifurcation case, (B); or transitional case, (A); and in all other cases it is unstable. 
Thus for an element $\varphi \in \mathscr{H}$ the stability properties are completely classified by the above scheme.

Of course the stability properties of many of these periodic points are already known. For hyperbolic points see [2], for general elliptic or $k$-bifurcation, $k \geqq 5$, see [4] and for extremal see [3]. The instability of a periodic solution of a time dependent Hamiltonian system of one degree of freedom in a case analogous to our 3-bifurcation point was established in [6]. Also see [5] for a discussion of LeviCivita's proof of the unstability of 3-bifurcation points. We shall consider this case directly and give a new proof via Liapunov functions. Thus the only cases considered here are 3-bifurcation points and the two cases under transitional and 4-bifurcation points.

3. The proof of Theorem 2.3. In order to establish Theorem 2.3 we need the following extension of Lemma 2.4 of [3].

Lemma 3.1. Let $l>8$ be an integer and $(x, s)$ a fixed point of $\varphi \in \mathscr{F}$. Then there exists a neighborhood $U$ of $(x, s)$ such that for each neighborhood $\mathscr{U}$ of $\varphi$ there is a $\psi \in \mathscr{U}$ and

(a) each fixed point of $\psi$ in $U$ is either hyperbolic, elliptic, extremal or transitional and

(b) each fixed point of $\psi$ in $U$ whose multipliers are $k$ th roots of unity $3 \leqq k \leqq l$ are $k$-bifurcation points and

(c) any other elliptic fixed point of $\psi$ in $U$ when written in the chart of $\$ 2$ has $\beta_{1}(0)^{2}+\beta_{2}(0)^{2} \neq 0$.

Moreover for each such $\psi$ there is a neighborhood $\mathscr{V}$ of $\psi$ such that for all $\zeta \in \mathscr{V}$ the fixed points of $\zeta$ in $U$ are as described in (a), (b), (c) above.

Proof. Clearly the only new part to prove is (c) and so we can assume the lemma with the exception of (c). Thus we need only consider the case when $(x, s)$ is a fixed point with multipliers $\exp ( \pm 2 \pi \omega i), \omega \neq a / b, 0<a<b \leqq 8$. Choose a chart $(q, p, \varepsilon)$ as described in $\S 2$ with domain $K \times L, K \subset M$ and $L \subset S$. Let $L^{\prime}$ be an open set with closure contained in the interior of $L$ and let $\delta: S \rightarrow R$ be a smooth function such that $\delta\left(L^{\prime}\right)=1$ and $\delta(S-L)=0$.

Consider the map $g: L \rightarrow R^{2}: \varepsilon \rightarrow\left(\beta_{1}(\varepsilon), \beta_{2}(\varepsilon)\right)$. By the elementary transversality theorem there exists a $\bar{g}$ arbitrarily close to $g$ on $L$ such that the image of $L$ by $\bar{g}$ does not meet $(0,0)$. Let $\bar{g}(\varepsilon)=\left(\bar{\beta}_{1}(\varepsilon), \bar{\beta}_{2}(\varepsilon)\right)$. The new map is given by $(\theta, r, \varepsilon) \rightarrow(\Theta, R)$ where

$$
\Theta=\theta+2 \pi \omega+\varepsilon \alpha(\varepsilon)+\delta(\varepsilon)\left\{\bar{\beta}_{1}(\varepsilon) r+\bar{\beta}_{2}(\varepsilon) r^{2}\right\}+(1-\delta(\varepsilon))\left\{\beta_{1}(\varepsilon) r+\beta_{2}(\varepsilon) r^{2}\right\}+\Theta_{1}(\theta, r \varepsilon) .
$$

The second part of the lemma is clear.

Now the proof of Theorem 2.3 proceeds in the same manner as the proof of Theorem 2.2 of [3] with the above lemma replacing Lemma 2.4.

4. Proof of Theorem 2.4. The next five lemmas analyze the stability properties of the remaining cases and thus constitute the proof of Theorem 2.4. 
LEMMA 4.1. The 3-bifurcation point is unstable.

Proof. Choose coordinates at the 3-bifurcation point as described in Lemma 1.14 of [3]. Since we are considering a fixed value of the parameter $s \in S$ it will be suppressed in the notation. Compute $\varphi^{3}:(\theta, r) \rightarrow\left(\Theta^{3}, R^{3}\right)$ where

$$
\Theta^{3}=\theta+\gamma(\cos 3 \theta) r^{1 / 2}+O(r), \quad R^{3}=r+2 \gamma(\sin 3 \theta) r^{3 / 2}+O\left(r^{2}\right) .
$$

Consider $V=(\sin 3 \theta) r^{3 / 2}$ and compute $\Delta V=v\left(\Theta^{3}, R^{3}\right)-V(\theta, r)=3 \gamma r^{2}+O\left(r^{5 / 2}\right)$. Thus we have a scalar function $V$ of variable sign whose difference $\Delta V$ is definite in sign and thus by the usual Liapunov argument the point is unstable. The details of such a proof are given in Proposition 1.11 of [3].

LemMA 4.2. The 4-bifurcation point is unstable if $|\beta|<|\gamma|$.

Remark. This is the same as case (A) in Proposition 1.17 of [3].

Proof. Proceed as in the previous lemma but let $V=(\sin 4 \theta) r^{2}$ and compute $\Delta V=4(\gamma+\beta \cos 4 \theta) r^{3}$.

LEMMA 4.3. The 4-bifurcation point is stable if $|\beta|>|\gamma|$.

REMARK. This corresponds to case (B) of Proposition 1.17 of [3].

Proof. Choose coordinates as before and compute $\varphi^{4}:(\theta, r) \rightarrow\left(\Theta^{4}, R^{4}\right)$ where

$$
\Theta^{4}=\theta+(\beta+\gamma \cos 4 \theta) r+O\left(r^{3 / 2}\right), \quad R^{4}=r+2 \gamma(\sin 4 \theta) r^{2}+O\left(r^{5 / 2}\right) .
$$

As the map stands the invariant curve theorem of Moser does not apply since the coefficient of $r$ in $\Theta^{4}$ depends on $\theta$. Make the change of coordinates $\theta=\varphi$ and $\rho=(\beta+\gamma \cos 4 \theta) r$ and compute $\varphi^{4}:(\varphi, \rho) \rightarrow(\Phi, P)$ where

$$
\Phi=\varphi+\rho+O\left(\rho^{3 / 2}\right), \quad P=\rho+O\left(\rho^{2}\right) .
$$

In these coordinates the invariant curve theorem can be applied to insure stability. Note that the invariant curve theorem as given in [4] does not require the map to be symplectic with the usual symplectic structure. It only requires that circles and their images intersect. Thus even though the above change of variable is not sympletic, the theorem can be applied in this case.

LEMmA 4.4. Transitional periodic points are unstable if $2 \eta+\alpha^{2}>0$.

Remark. This corresponds to case (B) of Proposition 1.13 of [3].

Proof. Make the change of scale $Q=\mu R_{1} P=\mu^{2} T, q=\mu r, p=\mu^{2} t$, where $\mu>0$. Compute $\varphi^{2}:(r, t) \rightarrow\left(R^{2}, T^{2}\right)$ where

$$
R^{2}=r-2 \mu\left\{t-\alpha r^{2} / 2\right\}+O\left(\mu^{2}\right), \quad T^{2}=t-2 \mu\left\{\alpha r t+\eta r^{3}\right\}+O\left(\mu^{2}\right) .
$$

Consider the Liapunov function $V=2 r t-\alpha r^{3}$ and compute

$$
\Delta V=V\left(R^{2}, T^{2}\right)-V(r, t)=-2 \mu\left(2 t^{2}-2 \alpha r^{2} t+\left\{2 \eta+3 \alpha^{2} / 2\right\} r^{4}\right)+O\left(\mu^{2}\right) .
$$

Which is negative definite for $\mu$ small enough. Note $\left(2 t^{2}+2 \alpha r^{2} t+\left\{2 \eta+3 \alpha^{2} / 2\right\} r^{4}\right)$ 
is a positive definite quadratic form in $t$ and $r^{2}$. Moreover the terms denoted by $O\left(\mu^{2}\right)$ are at least cubic in $t$ and $r^{2}$. Thus again the standard Liapunov argument establishes instability.

Lemma 4.5. Transitional periodic points are stable if $2 \eta+\alpha^{2}<0$.

Remark. This corresponds to case (A) of Proposition 1.13 of [3].

Proof. Start with the same set of coordinates as in the previous lemma. Change coordinates by $x=s^{1 / 2}\left(t-\alpha r^{2} / 2\right), \quad y=s^{1 / 2} r$ where $s=-\left(2 \eta+\alpha^{2}\right) / 4$ so that $\varphi^{2}:(x, y) \rightarrow(X, Y)$ where

$$
X=x+4 \mu y^{3}+O\left(\mu^{2}\right), \quad Y=y-2 \mu x+O\left(\mu^{2}\right) .
$$

Let $\varphi(\theta)=\sin$ lemn $\theta$, see Whittaker and Watson [7, p. 524], and let $\psi(\theta)$ $=(d / d \theta) \varphi(\theta)$. Change to the "polar coordinates" $(\rho, \theta)$ by $x=\rho^{2} \psi(\theta), y=\rho \varphi(\theta)$ and $X=P^{2} \psi(\Theta), Y=P \varphi(\Theta)$. Since $\varphi^{4}+\psi^{2} \equiv 1, \rho^{4}=x^{2}+y^{4}$ and $P^{4}=X^{2}+Y^{4}$,

$$
\begin{aligned}
P^{4}=X^{2}+Y^{4} & =\left(x+4 \mu y^{3}+O\left(\mu^{2}\right)\right)^{2}+\left(y-2 \mu x+O\left(\mu^{2}\right)\right)^{4} \\
& =x^{2}+8 \mu x y^{3}+O\left(\mu^{2}\right)+y^{4}-8 \mu x y^{3}+O\left(\mu^{2}\right)=\rho^{4}+O\left(\mu^{2}\right) .
\end{aligned}
$$

Thus $P=\rho+O\left(\mu^{2}\right)$.

Let $\Theta=\theta+\mu b+O\left(\mu^{2}\right)$ where $b$ is to be found.

$$
\begin{aligned}
Y & =P \varphi(\Theta)=\rho \varphi(\Theta)+O\left(\mu^{2}\right)=\rho \varphi(\theta)+\mu b \rho \psi(\theta)+O\left(\mu^{2}\right) \\
& =y+\mu x(b / \rho)+O\left(\mu^{2}\right) .
\end{aligned}
$$

By comparing with the above formula for $Y$ we see that $(b / \rho)=-2$ or $b=-2 \rho$. Thus $\varphi^{2}:(\theta, \rho) \varphi^{2}:(\theta, \rho) \rightarrow(\Theta, P)$ where $\Theta=\theta-2 \mu \rho+O\left(\mu^{2}\right)$ and the invariant curve theorem can now be applied to insure stability.

\section{REFERENCES}

1. G. D. Birkhoff, Dynamical systems, Amer. Math. Soc. Colloq. Publ., vol. 9, Amer. Math. Soc., Providence, R. I., 1927; rev. ed., 1966. MR 35 \#1.

2. P. Hartman, Ordinary differential equations, Wiley, New York, 1964. MR 30 \#1270.

3. K. R. Meyer, Generic bifurcation of periodic points, Trans. Amer. Math. Soc. 149 (1970), 95-107.

4. J. Moser, On invariant curves of area-preserving mappings of an annulus, Nachr. Akad. Wiss. Göttingen Math.-Phys. Kl. II, 1962, 1-20. MR 26 \#5255.

5. C. L. Siegel, Vorlesungen über Himmelsmechanik, Springer-Verlag, Berlin, 1956. MR 18, 178.

6. D. L. Slotnick, Asymptotic behavior of solutions of canonical systems near a closed, unstable orbit. Contributions to the theory of nonlinear oscillations. Vol. IV, Ann. of Math. Studies, no. 41, Princeton Univ. Press, Princeton, N. J., 1958, pp. 85-110. MR 21 \#166.

7. E. T. Whittaker and G. N. Watson, $A$ course in modern analysis, 4th ed., Cambridge Univ. Press, New York, 1940.

UNIVERSITY OF MINNESOTA, Minneapolis, Minnesota 55455 